\title{
Efficient generation of highly ionized calcium and titanium plasma columns for collisionally excited soft-x-ray lasers in a fast capillary discharge
}

\author{
J. J. Rocca, O. D. Cortázar, F. G. Tomasel, and B. T. Szapiro \\ Department of Electrical Engineering, Colorado State University, Fort Collins, Colorado 80523
}

(Received 10 June 1993)

\begin{abstract}
Fast discharges through 1.5-mm-diam capillaries have produced dense $\mathrm{Ca}$ and $\mathrm{Ti}$ plasma columns with an abundance of Ne-like ions, which are of interest for the development of small-scale, collisionally excited soft-x-ray lasers. Current pulses of $30 \mathrm{~ns}$ full width at half maximum and peak currents of less than $70 \mathrm{kA}$ produced plasmas with line emission from ions with charge up to the F-like state. Line emission at the wavelengths of the $3 p-3 s$ and $3 d-3 p$ transitions of the Ne-like ions has been observed.
\end{abstract}

PACS number(s): $52.80 .-\mathrm{s}, 42.55 . \mathrm{Vc}$

High-power lasers, of the type employed in inertialconfinement fusion research, have been successfully utilized to generate highly ionized plasmas with an abundance of Ne-like and Ni-like ions for soft-x-ray amplification $[1,2]$. Soft- $x$-ray lasing in collisionally excited Ne-like ions was demonstrated in these plasmas in several elements ranging from $\mathrm{Ti}(Z=22)$ to $\mathrm{Ag}(Z=47)$ $[1,2]$. Multiterawatt pulsed power machines producing current pulses in the range of mega amperes have been considered as alternative drivers for collisionally excited lasers [3]. However, the widespread use of soft-x-ray lasers in a number of interesting applications remains largely inhibited by the size, cost, and complexity of the soft-x-ray sources.

Recently, significant efforts have been devoted to the utilization of smaller laser drivers in the development of small-scale soft-x-ray lasers based on both collisional excitation and recombination laser schemes [4-6]. Gainlength products of 2.5 and 2 have been reported at 20.4 $\mathrm{nm}$ in $\mathrm{Ni}$-like $\mathrm{Nb}$ and at $18.2 \mathrm{~nm}$ in $\mathrm{CVI}$, respectively, utilizing table-top laser drivers [4,5]. A proposed alternative approach toward compact, efficient, and simpler soft-x-ray lasers consists in the direct generation of the amplifying plasma columns by a fast discharge through a capillary channel $[7,8]$. In this Rapid Communication we report the efficient generation of dense $\mathrm{Ca}$ and $\mathrm{Ti}$ plasma columns by a fast capillary discharge in which modest currents are required to reach the Ne-like ionization state. $\mathrm{Ca}$ and $\mathrm{Ti}$ plasmas in which atoms are ionized up to the F-like state were produced by currents of less than $70 \mathrm{kA}$ in $1.5-\mathrm{mm}$ capillaries. These capillary discharges are unique in generating elongated plasma channels with those characteristics, using such small currents. Emission from the $3 p-3 s$ and $3 d-3 p$ transitions of Ne-like ions in a capillary discharge plasma is reported.

Discharges in plastic and Teflon capillaries that produce plasmas, in which $\mathrm{C}, \mathrm{O}$, and $\mathrm{F}$ ions are the dominant species, have been studied as sources of continuum and soft $\mathrm{x}$ rays for spectroscopy, microscopy, and lithography [9], and, more recently, as possible plasmas for soft-x-ray recombination lasers [10]. However, the development of collisionally excited soft-x-ray lasers in the $\mathrm{Ne}$-like and $\mathrm{Ni}$-like sequences requires the generation of highly ionized plasma columns in heavier elements. Recently we reported the generation of hot $\left(T_{e}>150 \mathrm{eV}\right)$ dense Ar plasma columns of small diameter by fast discharge excitation of a preionized gas-filled capillary [8]. The creation of elongated capillary plasma columns in elements with $Z>18$ is of significant interest because the gain in the $3 p-3 s$ lines of Ne-like ions increases with the nuclear charge, having a maximum near Se $(Z=34)$ [11]. However, in this case the generation of capillary plasmas requires the development of a different approach, as, in contrast with Ar, these elements are solid at room temperature.

The $\mathrm{Ca}$ and $\mathrm{Ti}$ plasmas reported herein were produced by ablation of the walls of capillary channels perforated through insulating solid materials containing these elements. The fast current pulses rapidly detach the plasma from the walls, limiting both the amount of material ablated and the heat conduction losses, to form a hot, compressed plasma channel. Capillaries were made by binding calcium hydride powder, $\mathrm{CaH}_{2}$, with epoxy resin (80\%-20\% by weight). Similarly, the Ti plasmas were created by ablation of the walls of capillaries made out of titanium hydride, $\mathrm{TiH}_{2}$, and epoxy resin. Alternatively pure $\mathrm{CaH}_{2}$ capillaries, which do not contain resin, were developed by pressing $\mathrm{CaH}_{2}$ powder to $0.7 \mathrm{GPa}$. In all these capillary structures multiple discharge shots can be performed on a single capillary.

The capillary plasmas were excited by a fast, highvoltage pulse generator that produced current pulses with a $10-90 \%$ risetime of 13 ns and a full width at half maximum of $30 \mathrm{~ns}$ through a 1-cm-long capillary load. The pulse generator consisted of a 3-nF circular parallel-plate capacitor that, for the experiments reported herein, was charged by a six-stage, $V \leq 600 \mathrm{kV}$ Marx generator. A schematic diagram of the pulse generator and discharge configuration is shown in Ref. [11]. The capillary, which was placed in the axis of the capacitor, was evacuated to a pressure below $2 \times 10^{-5}$ Torr and then excited by discharging the capacitor through a low-inductance circuit that included the capillary channel and an $\mathrm{SF}_{6}$ pressurized spark-gap switch. The fast discharge pulse was preceded in a few microseconds by a preionization spark 
produced by discharging a small $(3 \mathrm{nF}, 12 \mathrm{kV})$ capacitor between a closely spaced third electrode and the anode electrode, with the purpose of enhancing the uniformity of the breakdown, and it was immediately preceded by a discharge prepulse having a current of approximately 1 $\mathrm{kA}$ and a duration of $50 \mathrm{~ns}$.

The axial soft-x-ray emission from the capillary plasmas was focused by a grazing-incidence mirror into the slit of a grazing-incidence spectrograph. The first part of the study, consisting in the determination of the degree of ionization of the plasma as a function of current and the study of its temporal evolution, was conducted with a 1-m spectrograph utilizing either a 600 - or a 1200 lines $/ \mathrm{mm}$ gold-coated grating blazed at $1^{\circ}$ and mounted at an angle of incidence of $86^{\circ}$. The identification of the $3 p-3 s$ transitions of $\mathrm{Ne}$-like species, which required increased spectral resolution, was accomplished utilizing a 2.2-m grazing-incidence spectrograph provided with a 1200 lines $/ \mathrm{mm}$ gold-coated grating set at $85.8^{\circ}$. In both instruments the detection system consisted of a multichannel-plate intensifier (MCP), a phosphorous screen, and a linear diode array detector. Time-resolved spectra with approximately 5-ns resolution were obtained by gating the MCP with a high-voltage pulse.

The soft-x-ray emission from $\mathrm{Ca}$ capillary plasmas is discussed next. Figure 1 shows the time-resolved spectra, obtained at $6 \mathrm{~ns}$ after the peak of the current pulse, of the
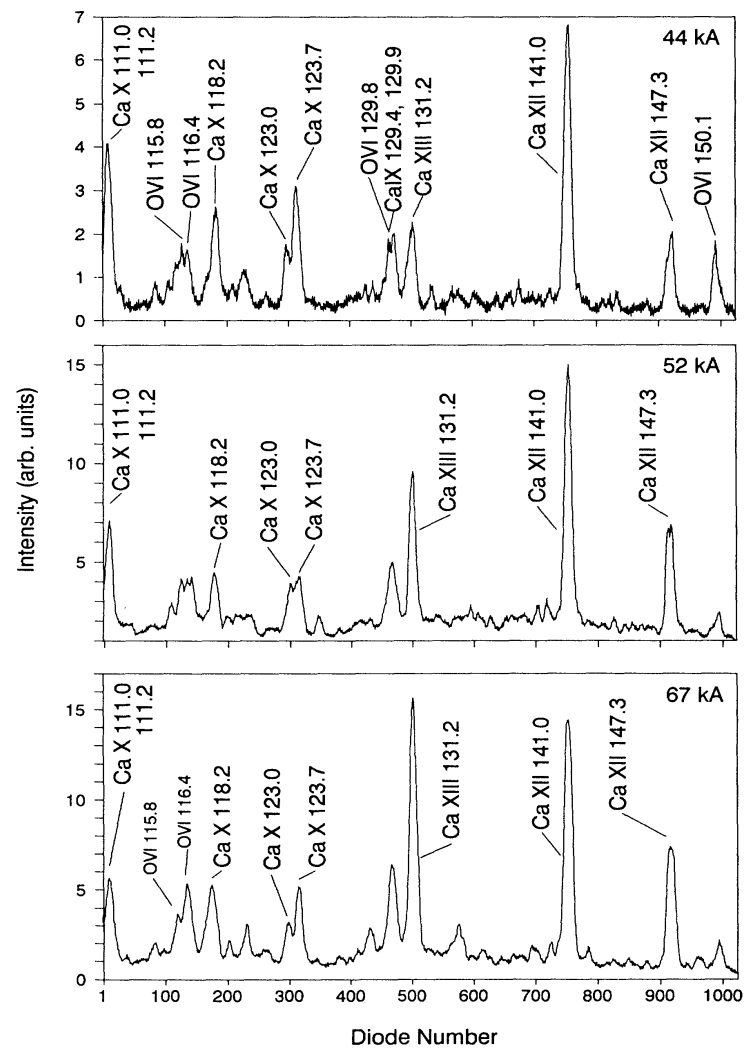

FIG. 1. Time-resolved spectra $(110-150 \AA$ ) from a $1.5-\mathrm{mm}$ diam, 1-cm-long $\mathrm{CaH}_{2}$ capillary corresponding to three different values of the discharge current pulse. soft-x-ray emission from Ca plasmas generated in a 1.5mm-diam, $1-\mathrm{cm}$-long capillary by current pulses of three different amplitudes. For the 44-kA discharge the most intense lines in this spectral region correspond to Na-like $\mathrm{Ca}, \mathrm{Ca} \mathrm{X}$, and to F-like $\mathrm{Ca}, \mathrm{Ca} \mathrm{XII}$, an indication that in a $1.5-\mathrm{mm}$-diam capillary this relatively small current is sufficient to generate $\mathrm{Ne}$-like $\mathrm{Ca}$ ions. Ca IX and Ca XIII lines are also observed. As the current increases, the plasma becomes more ionized, as shown by the increase in intensity of the $\mathrm{Ca}$ XIII lines and by the relative decrease of the $\mathrm{CaX}$ lines. At a current of $67 \mathrm{kA}$ the Ca XIII 131.2-nm line dominates the spectrum. These results show that the degree of ionization of these plasmas can be selected by adjusting the magnitude of the current pulse. Similar spectra were obtained with capillaries of the same diameter and length of 3 and $5 \mathrm{~cm}$, by increasing the discharge voltage to compensate for the decrease in discharge current caused by the larger inductance associated with a longer capillary.

Figure 2 is a sequence of time-resolved spectra displaying the temporal evolution of the soft-x-ray emission for a 65-kA discharge through a $\mathrm{CaH}_{2}$ capillary. The time of each spectrum relative to the current pulse is indicated. In the first spectrum, obtained at $8 \mathrm{~ns}$ after the initiation of the current pulse, only $\mathrm{Ca}$ IX and $\mathrm{CaX}$, as well as $\mathrm{O}$
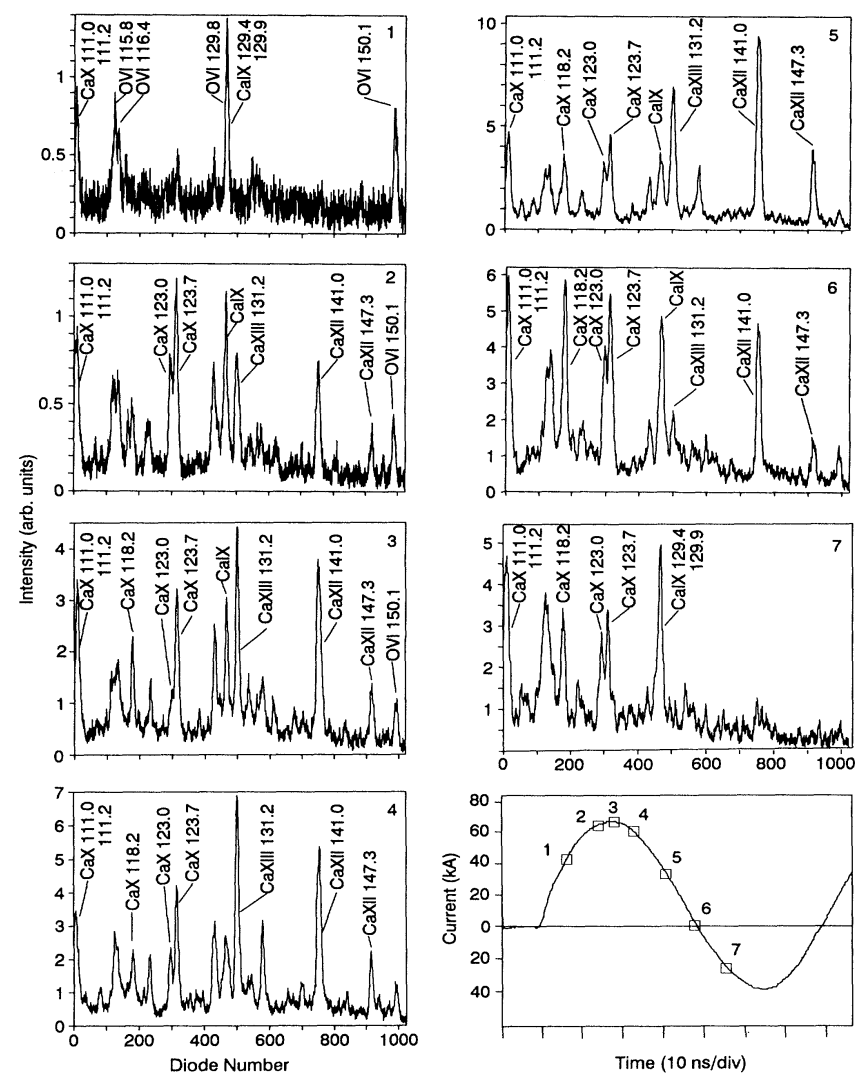

FIG. 2. Sequence of time-resolved spectra (110-150 $\AA$ ) from a 1.5-mm-diam, 1-cm-long capillary containing $\mathrm{CaH}_{2}$ excited by a 65-kA current pulse. The time at which each spectra was obtained with respect to the discharge current pulse is indicated. 
impurity lines, are observed. The degree of ionization of the plasma increases to reach a maximum shortly after the peak of the current pulse, when Ca XIII emission is observed. By the time of the end of the first half-cycle of the current pulse the Ca XIII line has practically disappeared, and again $\mathrm{Ca} I \mathrm{X}$ and $\mathrm{CaX}$ lines dominate the spectra. During the second half-cycle of the current pulse the plasma continues to cool, as more ablated material from the capillary walls is injected into the plasma column.

Discharges in $\mathrm{TiH}_{2}$ capillaries produced plasma containing highly ionized Ti ions. Figure 3 shows a timeresolved spectrum covering the $97-134-\AA$ region corresponding to a $61-\mathrm{kA}$ discharge through a $1.5-\mathrm{mm}$-diam, 3-cm-long capillary containing $\mathrm{TiH}_{2}$. Line emission from Na-like Ti, Ti XII, and F-like Ti, Ti XIV, is observed. Other $\mathrm{Ti}$ transitions, as well as chlorine lines corresponding to ionization states ranging from $\mathrm{Cl} I X$ to $\mathrm{Cl}$ XIII, have also been identified in the spectra of these capillaries in other wavelength regions. $\mathrm{Cl}$ is found as an impurity in the epoxy resin used to bind the $\mathrm{TiH}_{2}$. Due to the relatively low $\mathrm{Cl}$ concentration, approximately $300 \mathrm{ppm}$, most of these lines can be expected to be optically thin, and therefore constitute a good diagnostic tool. The ratio of intensities of the $\mathrm{ClX}$ resonant lines was verified to be very close to the optically thin value. An estimate of the electron density was obtained from the line intensity ratio of selected $\mathrm{Cl}$ XI lines that are sensitive to the electron density and relatively insensitive to variations in the electron temperature [12]. The intensity ratio of $\mathrm{ClXI}$ $2 s^{2} 2 p^{32} D_{3.2}^{o}-2 s 2 p^{42} D_{3 / 2} \quad(209.18 \AA)$ and $2 s 2 p^{42} D_{5.2}-$ $2 p^{52} P_{3 / 2}^{o}(195.69 \AA)$ lines, selected for this purpose, was computed as a function of the plasma density from the relative populations of their upper levels resulting from solving a set of rate equations for the $2 p^{5}, 2 s 2 p^{4}$, and $2 s^{2} 2 p^{3}$ levels. The collisional and radiative rates coupling the population of these levels were computed utilizing the transition probabilities given by Cheng, Kim, and Desclaux [13], assuming an electron temperature of $150 \mathrm{eV}$. The majority of a set of 15 spectra obtained $8 \mathrm{~ns}$ after the peak of the current pulse for excitation currents between 40 and $66 \mathrm{kA}$ yielded line intensity ratios that correspond to densities in the range of $4 \times 10^{18}$ to $2 \times 10^{19} \mathrm{~cm}^{-3}$.

The $3 p-3 s$ laser candidate transitions and the $3 d-3 p$ lines in the Ne-like ions are normally very weak, but they have been previously identified in spectra from laser-

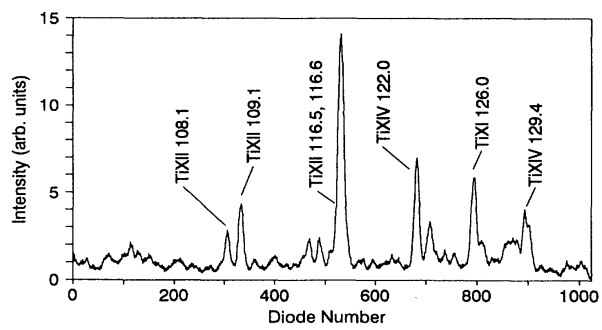

FIG. 3. Time-resolved spectrum from a 1.5-mm-diam, 3-cmlong capillary containing $\mathrm{TiH}_{2}$. The spectrum was obtained 10 ns after the peak of a $61-\mathrm{kA}$ current pulse. created plasmas and beam-foil experiments [14,15]. In Ca XI some of the $3 p-3 s$ transitions fall in between the strong $\mathrm{Ca} X$ resonant lines at $557.74 \AA$ and $574.01 \AA$, which provide for a convenient wavelength calibration. The calibration of this spectral region was completed utilizing the Ar II lines from a low-pressure dc discharge. Figure 4(a) shows an average of 14 spectra in this wavelength region, corresponding to $\mathrm{CaH}_{2}$ capillaries $1.5 \mathrm{~mm}$ in diameter and $3 \mathrm{~cm}$ in length, obtained $8 \mathrm{~ns}$ after the peak of current pulses of the order of $50 \mathrm{kA}$. Lines were observed at $565.40 \pm 0.15 \AA$ and $567.10 \pm 0.15 \AA$, which correspond well with the wavelengths of the $3 s{ }^{1} P_{1}-3 p{ }^{3} P_{2}(565.32 \AA)$ and $3 s{ }^{3} P_{2}-3 p{ }^{3} D_{3}(566.98 \AA)$ lines of Ne-like $\mathrm{Ca}$ [14]. The first of these lines, the $J=2-J=1$ transition, is a candidate for amplification. Also, spectra obtained in the wavelength region corresponding to the three $3 d-3 p$ Ca XI transitions $3 p^{3} D_{2}-3 d^{3} F_{3}\left(427.05 \AA\right.$ ), $3 p{ }^{3} P_{2}-3 d^{3} D_{3}(431.50 \AA)$ and $3 p{ }^{3} D_{3}-3 d{ }^{3} F_{4}$ (435.00 ̊) [14], two of which contribute to populate the upper levels of the above-mentioned $3 p-3 s$ lines by radiative cascade, show lines whose wavelengths agree to within $0.1 \AA$ with those of the above-mentioned $3 d-3 p$ transitions.

Of greater interest is the identification of the $J=0-J=1$ lines, which have been observed for low $-Z$ elements to have the largest gain in laser-created plasmas [2], in agreement with calculations. Wavelength calibration in the spectral region corresponding to the $3{ }^{1} P_{1}-{ }^{3} P{ }^{1} S_{0}$ Ca XI transition was obtained utilizing Ca IX lines from the same discharge, as well as the $3 d-2 p$

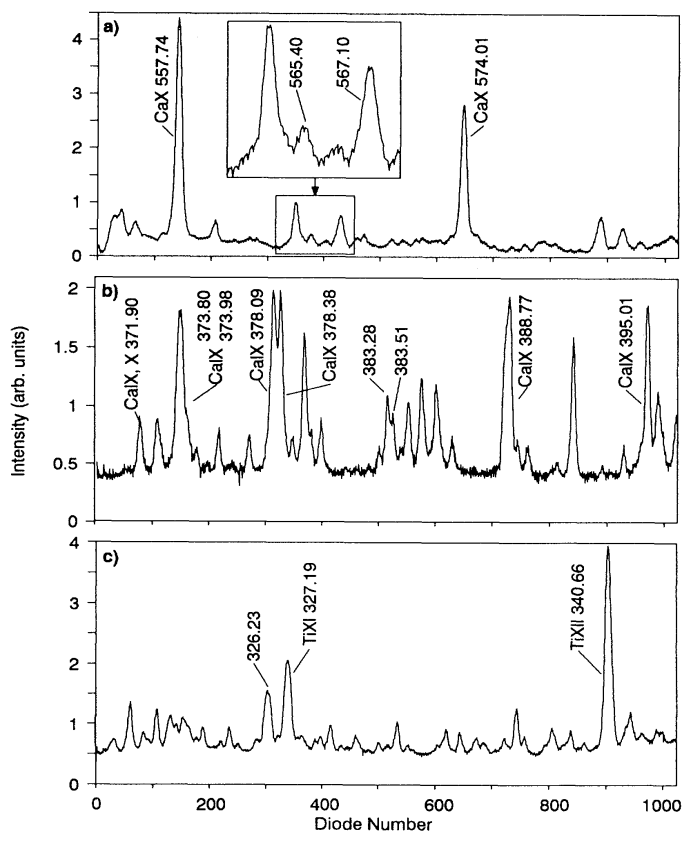

FIG. 4. (a) Average of 14 spectra from compressed $\mathrm{CaH}_{2}$ capillaries, covering the region between the strong 557.74- and 574.01- $\AA$ resonant lines of $\mathrm{CaX}$. (b) Spectrum from a compressed $\mathrm{CaH}_{2}$ capillary in the neighborhood of $383 \AA$. (c) Spectrum from capillary containing $\mathrm{TiH}_{2}$. 
C IV lines from a low-current polyethylene discharge and $\mathrm{Na}$ VII lines. The major impurity in the $\mathrm{CaH}_{2}$ is known to be $\mathrm{O}$, and an analysis of the material performed to identify heavy metal impurities found only $\mathrm{Mg}$ and $\mathrm{Al}$ at concentrations greater than $0.01 \mathrm{ppm}$. None of these elements has known lines in the vicinity of $383.3 \AA$. The spectrum of Fig. 4(b), which corresponds to a 56-kA discharge through a $2.5-\mathrm{mm}$ capillary made out of pure $\mathrm{CaH}_{2}$, shows a line at $383.28 \pm 0.10 \AA$ and a wavelength which is in good agreement with the only previous measurement of the $3 s{ }^{1} P_{1}-3 p{ }^{1} S_{0}$ transition of Ca XI (383.27 A) [14] in a laser-created plasma. Also, as previously observed by Ragozin [16], this $J=0-1$ laser candidate transition appears closely spaced from a line at $383.51 \AA$. In our spectra these two transitions, which were also observed in $1.5-\mathrm{mm}$-diam capillaries, often appeared blended into a single feature at approximately $383.4 \AA$, having an intensity up to that of the strong Ca IX neighboring lines [17]. Experiments were also conducted to identify its isoelectronic equivalent in $\mathrm{Ti}$.

For this purpose, spectra covering the vicinity of $326 \AA$ were obtained from $\mathrm{TiH}_{2}$ capillary discharges. This region of the spectra was calibrated using P XI and P XII lines. Figure 4(c) shows a time-resolved spectrum from a 3-cm-long, 1.5-mm-diam $\mathrm{TiH}_{2}$ capillary discharge excited by a 50-kA current pulse. The line observed in this spectrum at $326.23 \pm 0.10 \AA$ corresponds well with the calculated (326.24 $\AA$, Ref. [2]) and previously measured wavelength (326.29 $\pm 0.05 \AA$, Ref. [15]; 326.3 $\pm 0.5 \AA$, Ref. [2]) of the $J=0-J=1$ transition of Ne-like Ti. It should be noticed nevertheless that in this case the most accurate measurement of this Ti XIII line, a beam-foil experiment
(Ref. [15]), inferred the contribution of a blended line at $326.1 \AA$ that could also be contributing to our spectra. The other two clearly observed lines in the spectrum of Fig. 4(c) are assigned to the Ti XI $327.19-\AA$ and the Ti XII 340.66- $\AA$ transitions.

In conclusion, we have generated dense, highly ionized $\mathrm{Ca}$ and $\mathrm{Ti}$ plasma columns, using a fast capillary discharge and identified line emission at wavelengths corresponding to the $3 p-3 s$ and $3 d-3 p$ lines in the Ne-like ions. The relatively small currents required to ionize the atoms to the Ne-like state could potentially lead to small-scale, discharge-pumped ultrashort-wavelength lasers by collisional excitation. Similar discharges in capillaries containing elements of the next row of the periodic table are expected to produce highly ionized plasma columns containing large concentrations of $\mathrm{Ni}$ like ions, which will allow for the exploration of gain in this sequence. Ni-like ions, which have the advantage of requiring a reduced excitation energy for lasing in a given wavelength range, and which have been predicted [18] to scale favorably to low $Z$, constitute very attractive candidates for lasing in a capillary discharge.

We acknowledge the collaboration of $\mathrm{Mr}$. Ken Richardson and Dr. Mario Marconi. We thank Dr. E. N. Ragozin for providing us with copies of his lasercreated-plasma Ca spectra, and Dr. V. N. Shlyaptsev and Professor Siu Au Lee for helpful discussions. This work was supported by the U.S. Department of Energy, Advanced Energy Project, Grant No. DE-F0291ER 12110 and by the National Science Foundation, Grant No. ECS9013372.
[1] D. L. Matthews et al., Phys. Rev. Lett. 54, 110 (1985); B. J. MacGowan et al., J. Appl. Phys. 61, 5423 (1983); T. N. Lee, E. A. McLean, and R. C. Elton, Phys. Rev. Lett. 39, 1185 (1987); T. Boehly et al., Phys. Rev. A 42, 6962 (1990); D. J. Fields et al., ibid. 46, 1606 (1992).

[2] J. Nilsen, B. J. MacGowan, L. B. Da Silva, and J. C. Moreno, Lawrence Livermore National Laboratory, Report No. UCRL-JC-112704 (1993).

[3] J. Davis, R. Clark, J. P. Apruzese, and P. C. Kepple, IEEE Trans. Plasma Sci. PS-16, 482 (1988).

[4] S. Basu, P. L. Hagelstein, M. Muendel, J. GoodBerlet, and S. Kaushik (unpublished).

[5] D. E. Kim, C. A. Skinner, D. Voorhees, and S. Suckewer, Proc. SPIE 1551, 295 (1991).

[6] T. Hara, K. Ando, H. Yashiro, and Y. Aoyagi, Jpn. J. Appl. Phys. 28, L1010 (1989).

[7] J. J. Rocca, D. C. Beethe, and M. C. Marconi, Opt. Lett. 13, 565 (1988).

[8] J. J. Rocca, O. D. Cortázar, B. Szapiro, K. Floyd, and F. G. Tomasel, Phys. Rev. E 47, 1299 (1993).

[9] H. Conrads, Z. Phys. 444, 200 (1967); P. Bogen, H. Conrads, G. Gatti, and W. Kohlhaas, J. Opt. Soc. Am. 58, 203 (1968); S. M. Zakharov, A. A. Kolomenski, S. A. Pikuz, and A. I. Samokhin, Pis'ma Zh. Tekh. Fiz. 6, 1135 (1980)
[Sov. Tech. Phys. Lett. 6, 486 (1980)]; R. A. McCorkle, Appl. Phys. A 26, 261 (1981).

[10] C. Steden and H.-J. Kunze, Phys. Lett. A 151, 1534 (1990); J. J. Rocca, M. C. Marconi, B. T. Szapiro, and J. Meyer, Proc. SPIE 1551, 275 (1991); C. A. Morgan, H. R. Griem, and R. C. Elton (unpublished); J. J. Rocca, M. C. Marconi, and F. G. Tomasel, IEEE J. Quantum Electron. 29, 182 (1993).

[11] A. V. Vinogradov and V. N. Shlyaptsev, Kvant. Elektron. (Moscow) 10, 2325 (1983) [Sov. J. Quantum Electron. 13, 1511 (1983)]; B. L. Whitten, R. A. London, and R. S. Walling, J. Opt. Soc. Am. B 5, 2537 (1988).

[12] U. Feldman and G. A. Doschek, J. Opt. Soc. Am. 67, 726 (1977).

[13] K. T. Cheng, Y.-K. Kim, and J. P. Desclaux, At. Data Nucl. Data Tables 24, 111 (1979).

[14] E. N. Ragozin, S. S. Churilov, E. Ya. Kononov, A. N. Ryabtsev, and Yu.F. Zayikin, Phys. Scr. 37, 742 (1988).

[15] E. Träbert and C. Jupén, Phys. Scr. 36, 586 (1987).

[16] E. N. Ragozin, private communication.

[17] J. J. Rocca, O. D. Cortázar, B. T. Szapiro, F. G. Tomasel, and D. Hartshorn, Proc. SPIE (to be published).

[18] P. L. Hagelstein, Proc. SPIE 1551, 254 (1991). 